\title{
Impact of atrial fibrillation awareness campaigns: interrupted time series using the Clinical Practice Research Datalink
}

\author{
Victoria Allan*,1, Cormac J Sammon², Laura McDonald', Faisal Mehmud ${ }^{3}$, Raza Alikhan ${ }^{4}$ \& \\ Sreeram V Ramagopalan ${ }^{1}$ \\ ${ }^{1}$ Centre for Observational Research \& Data Sciences, Bristol-Myers Squibb, Uxbridge, UB8 1DH, UK \\ ${ }^{2}$ PHMR Ltd, Berkeley Works, Berkley Grove, London, NW1 8XY, UK \\ ${ }^{3}$ Bristol-Myers Squibb, Uxbridge, UB8 1DH, UK \\ ${ }^{4}$ Department of Haematology, Cardiff \& Vale University Health Board, University Hospital of Wales, Cardiff, Wales, UK \\ *Author for correspondence: Tel.: +44 189552 3016; victoria.allan@bms.com
}

\begin{abstract}
Aim: This study investigated whether the rates of atrial fibrillation (AF) consultations changed following AF awareness campaigns in England. Materials \& methods: Among adults in the Clinical Practice Research Datalink, Poisson regression was used to model weekly rates of AF-related consultations over time. The models were used to assess whether rates changed in the 8 weeks following World Heart Rhythm Week (WHRW) and Global AF aware week. Results: A higher incidence of pulse checks was observed following WHRW (IRR 1.16 [95\% Cl 1.08-1.24]). No difference in the incidence of AF diagnoses was noted following WHRW (IRR: 1.03 [95\% Cl: 0.97-1.09]) or Global AF aware week (IRR: 0.94 [95\% Cl: 0.88-1.00]). Conclusion: The results suggest AF campaigns may increase awareness but do not bring about short-term increases in the rates of AF diagnoses.
\end{abstract}

First draft submitted: 10 May 2019; Accepted for publication: 10 June 2019; Published online: 26 June 2019

Keywords: clinical practice research datalink • database $\bullet$ general practice $\bullet$ health promotion $\bullet$ nonvalvular atrial fibrillation

Individuals with undiagnosed atrial fibrillation (AF) are unknowingly at an increased risk of experiencing fatal and disabling strokes but are not afforded the protection of preventative oral anticoagulants. As many as one in four ischemic stroke or transient ischemic attack survivors receive a subsequent first diagnosis of AF, as estimated in a recent meta-analysis of 50 studies [1]. Early diagnosis of AF is crucial for timely stroke prevention, yet AF is asymptomatic in a third of cases, which makes diagnosis challenging [2]. Systematic screening to detect AF is associated with a $57 \%$ increased odds of identifying cases, as compared with routine care, but has a higher cost burden [3]. The European Society of Cardiology therefore recommends opportunistic screening for AF in patients aged 65 years and older using pulse palpation, followed by confirmation of the diagnosis based on electrocardiogram (ECG) examination [4].

In the absence of systematic population-based screening programs, AF awareness campaigns, such as the annually held, World Heart Rhythm Week (WHRW) and Global AF aware week (GAFAW), may instead help to accelerate the diagnosis of $\mathrm{AF}$ [5]. AF awareness campaigns seek to educate both the general public and healthcare professionals to recognize breathlessness and palpitations as symptoms for AF as well as teaching the importance of regularly carrying out pulse checks. As reported in a recent cross-sectional survey across ten countries, there is low awareness of AF in the general population (only 48\% of 6312 survey respondents) [6], highlighting the need for educational campaigns [7]. Yet to date, there are no published quantitative evidence to suggest that campaigns, such as WHRW, lead to increases in AF-related healthcare consultations and ultimately to the diagnosis of new AF cases, nor is it clear whether campaigns have an overall population impact or only impact certain groups of people.

This study therefore aimed to investigate whether rates of new diagnoses of AF and select AF-related symptoms, diagnostic tests and prescriptions recorded in primary care in the UK changed in the 8 weeks following WHRW and GAFAW in the years 2012-2017. A clearer understanding of whether existing efforts to raise awareness about AF

Future Medicine 
are working can help inform future public health strategies for identifying undiagnosed AF and channel resources into targeting specific groups.

\section{Methods}

Study design \& setting

An interrupted time series (ITS) study design, leveraging longitudinal electronic medical records from the Clinical Practice Research Datalink (CPRD), was used to assess the short-term impact of WHRW and GAFAW on rates of AF-related consultations in UK primary care in the years 2012-2017. The CPRD contains a nationally representative subset of the UK population and includes records for diagnoses, symptoms, diagnostic tests and prescriptions, which are coded using the Read classification system [8]. A comprehensive tutorial on ITS methodology and applications in assessing effectiveness of public health interventions is provided by Bernal and colleagues [9]. In brief, a regression-based model is used to test the association between a longitudinal outcome measure (i.e., weekly rates of new AF diagnoses) and an exposure at a known point in time (i.e., WHRW), under the assumption that in the absence of the exposure, the trend in observing the outcome would continue unchanged. The postintervention impact (i.e., how WHRW affects AF event rates) was hypothesized to result in a temporary, short-term level change lasting 8 weeks before returning to the pre-intervention trend (conceptual diagram, Supplementary Figure 1).

\section{Study population}

The study population included all adults (aged 18 years and over) at risk of receiving a first diagnosis of AF (no prior Read codes for AF) with a minimum of 1 year of baseline data (continuous patient registration at a CPRD practice). Individuals entered the analysis on the earliest date during the study period in which inclusion criteria were fulfilled, if all criteria were fulfilled prior to the start of the study period patients entered at the start of the study period. Individuals exited the analysis on the earliest date of the date of deregistration with their general practice (GP), AF diagnosis, death, end of study period or last date of data collection from a patients GP.

\section{Exposure}

The exposures in the analysis were WHRW and GAFAW [5,10]. WHRW raises awareness about the importance of checking your heart rhythm, its relationship with arrhythmias and the symptoms and morbidity associated with arrhythmias. GAFAW appears to have a slightly narrower remit, focusing specifically on raising awareness about AF, the relationship between heart rhythm and AF, and the symptoms, diagnosis and morbidity associated with AF. As $\mathrm{AF}$ is the most prevalent arrhythmia there is significant overlap in the remit of the campaigns and at present a large amount of promotional materials (e.g., related to pulse checks) are shared by the campaigns. WHRW occurred in the 21 st, 22nd or 23rd week of the year during the study period. GAFAW occurred in the 47th week of years 2013 to 2015 of the study period. As outlined above, we tested an immediate, short-term effect of these awareness campaigns on our outcomes lasting 8 weeks, the week of the campaign and the subsequent 7 weeks.

\section{Outcomes}

The primary outcome was atrial fibrillation diagnoses, identified using a comprehensive list of Read codes. AF diagnoses in CPRD have been validated in a prior study reporting a positive predictive value of $96 \%$ [11]. Secondary outcomes were defined using codes indicative of tests used to identify atrial fibrillation (pulse checks, ECG tests), symptoms of AF (breathlessness, chest pain, palpitations), cardiovascular referrals and AF-related prescriptions (oral anticoagulants, parenteral anticoagulants, antiplatelets, antiarrhythmics, etc).

\section{Statistical analysis}

The frequency and rate of the primary outcome and each secondary outcome were calculated in each calendar week over the study period 1 January 2012 and 31 December 2017. Two parallel analyses were performed, one in which each week in the study period was stratified according to whether patients were exposed to WHRW campaign and the other in which each week in the study period was stratified according to whether patients were exposed to GAFAW.

The ITS analyses utilized Poisson regression to model the number of postexposure outcome events occurring during the week of the campaign or in the subsequent 7 weeks. The total person time contributed by the study population was included in the model as an offset variable, thereby converting the outcome to a rate in order to adjust for changes in the study population over time [9]. Common sources of bias in ITS analyses include seasonal 
Table 1. Characteristics of the study population at study entry.

\begin{tabular}{|ll|}
\hline Characterstic & $n(\%)$ \\
\hline Total & $5,902,048(100)$ \\
\hline Sex: & \\
\hline - Male & $2,914,316(49)$ \\
\hline- Female & $2,987,732(51)$ \\
\hline Age (years): & $4,651,321(79)$ \\
\hline$-0-64$ & $596,054(10)$ \\
\hline$-65-74$ & $380,208(6)$ \\
\hline$-75-84$ & $274,465(5)$ \\
\hline$-85-120$ & \\
\hline
\end{tabular}

variation in observing outcome events and correlation between consecutive points in the time series. Seasonality adjusted models were developed by including Fourier terms in the Poisson model. The Fourier terms are pairs of sine and cosine functions of time with an underlying period reflecting the seasonal cycle [12]. Autocorrelation was assessed through examining plots of residuals and partial autocorrelation functions.

Effect modification was explored via subgroup analyses on age $(<65,65-74,75-84,85-120$ years) and sex (male/female). Sensitivity analyses were used to explore the impact of using alternative risk periods post awareness campaign (WHRW/GAFAW).

All analyses were carried out using Stata/IC version 15.1.

The public/patients were not involved in setting the research question or the outcome measures, developing plans for design or conduct of the study or interpreting the results. There are no immediate plans to disseminate the results to the patient community.

\section{Results}

We identified 5,902,048 patients meeting the study inclusion criteria. Table 1 describes the sex and age distribution of the study population at the start of patient follow-up.

For WHRW and GAFAW, and for both the primary outcome and each of the secondary outcomes, Figure 1 displays the frequency of events in the baseline and post-awareness campaign periods, along with seasonality adjusted IRRs from the Poisson regression models comparing the event rates in these periods. This information is provided alongside the crude incidence rates and IRRs in Supplementary Table 1. No difference in the rate of atrial fibrillation diagnoses was observed in the 8 weeks following the start of the GAFAW or WHRW campaigns (Figures $1 \& 2$ ). No difference was also observed when atrial flutter events were combined with atrial fibrillation events and when atrial fibrillation events are stratified into those with a stroke event recorded in the 60 days either side of the diagnosis and those without such diagnoses (Figure 1).

After adjusting for seasonal trends, the rate of pulse checks was $16 \%$ higher in the 8 -week periods following the WHRW campaigns than in the baseline periods ( IRR $_{\text {adj }}: 1.16$ [95\% CI: 1.08-1.24]); however, no such increase was noted in the 8-week period following GAFAW (IRR ${ }_{\text {adj }}$ : 0.88 [95\% CI: 0.82-0.94]; Figures $1 \& 2$ ). After adjusting for seasonality, no significant differences were noted in the rate of irregular pulse records or ECG events following WHRW. The rate of ECG tests was also no different following GAFAW; however, the rate of irregular pulse records was lower following GAFAW (Figure 1).

When investigating symptoms, after adjusting for seasonality the rate of breathlessness records was slightly higher following WHRW but a statistically significant difference in the rates of palpitations and chest pain records were not observed. In the 8 weeks following GAFAW, the rate of breathlessness, palpitations and chest pain events was $11 \%$ lower than in the baseline periods (Figure 1). The rate of cardiovascular referrals was also noted to be slightly higher following WHRW and slightly lower following GAFAW (Figure 1). After adjusting for seasonal trends, no notable differences in the rate of prescriptions were noted following WHRW or GAFAW (Figure 1).

Analyses exploring the sensitivity of results to the duration of the post awareness campaign risk periods are presented in Supplementary Figure 2. The statistically significant increase in the rate of pulse checks following WHRW was observed when the risk period was extended to 20 weeks but was not when it was reduced to 4 weeks. Subgroup analyses investigating the rates across age and sex did not identify any notable trends across these groups (Supplementary Figure 3). 


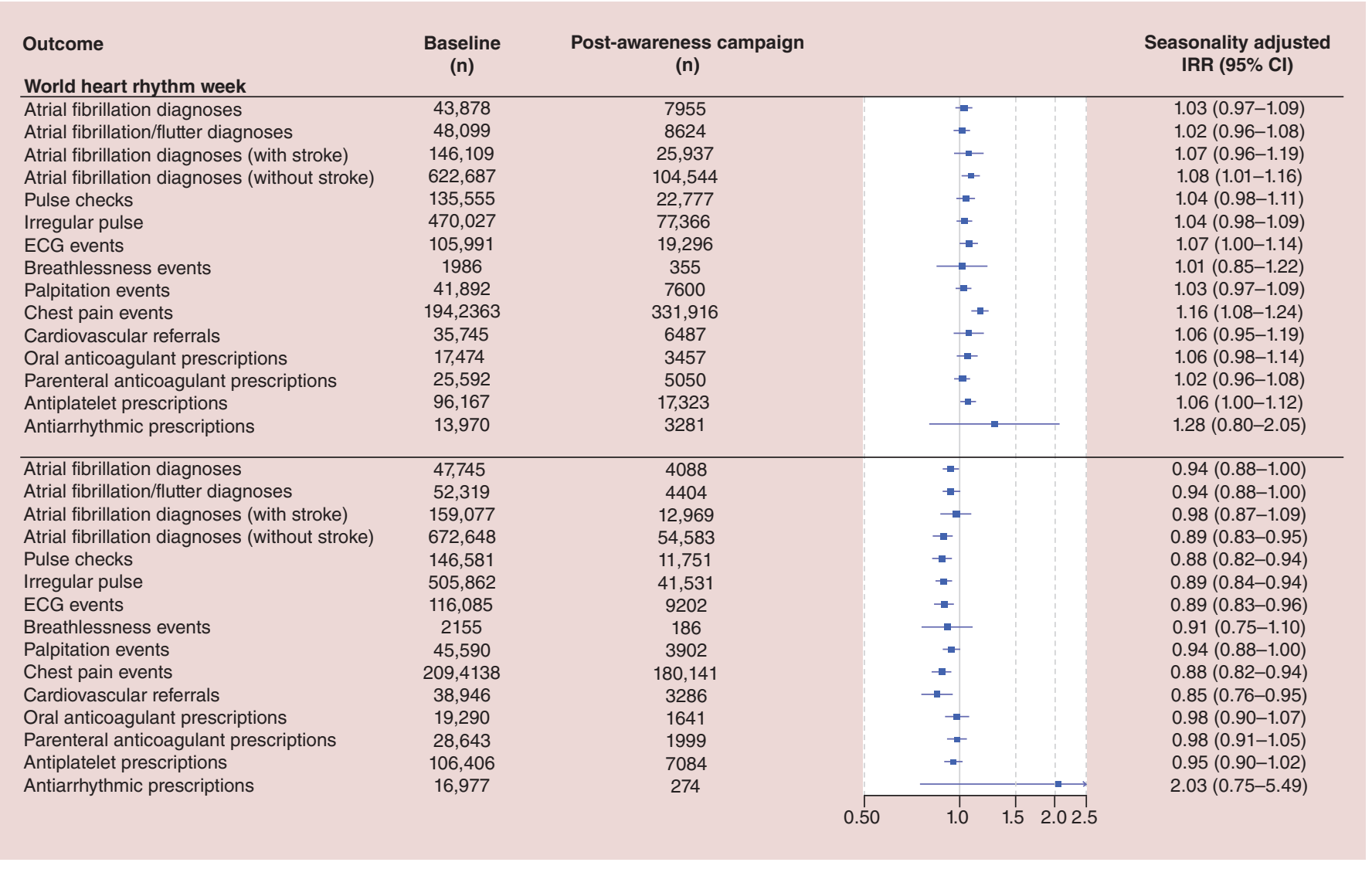

Figure 1. Forest plot showing the frequency of events in the baseline and post-awareness campaign (World Heart Rhythm Week and Global AF aware week) periods, along with seasonality adjusted IRRs obtained from Poisson regression models comparing the rates of events between baseline and post-awareness campaign periods.

ECG: Electrocardiogram; IRR: Incidence rate ratio.

\section{Discussion}

We identified no immediate impact of WHRW and GAFAW on the rate of atrial fibrillation diagnoses in the 8 weeks following these awareness campaigns. However, the rate of pulse checks was observed to be $16 \%$ higher in the 8 weeks following WHRW than in other weeks suggesting the campaign may be effective in increasing awareness about the importance of regular pulse checks.

The validity of ITS studies is highly dependent on the specification of an appropriate impact model and is therefore best suited to investigating interventions that occur at a single point in time and after which a permanent or well-defined temporary impact can be expected. As such, they are an important analytic tool in investigating the impact of one-off public health interventions. The choice of a model to describe the impact of the awareness campaigns under study was complicated by uncertainty regarding the expected impact of the awareness campaign and by its cyclical, recurring nature. We therefore chose to test the hypothesis that exposure to the WHRW and GAFAW campaigns led to an immediate, temporary (8 week) surge in patient-clinician consulting behaviors related to AF. Our inability to identify an impact of WHRW and GAFAW on the rate of AF diagnoses may be related to a misalignment between our hypothesis and the true model by which these campaigns might impact diagnosis rates. We sought to explore this via sensitivity analyses altering the duration of the impact period associated with the awareness campaign to varying periods between 4 and 20 weeks long; these suggested that the choice of a smaller or larger risk period would not have resulted in identification of an increase in AF diagnoses. However, it is possible that these campaigns had a longer term, sustained impact on diagnosis rates which we would not have been able to detect using our chosen impact model. Were this the case, the campaigns may contribute to the overall increase in the incidence rate of AF diagnoses we observe over calendar time in this study. We did not seek to assess the 

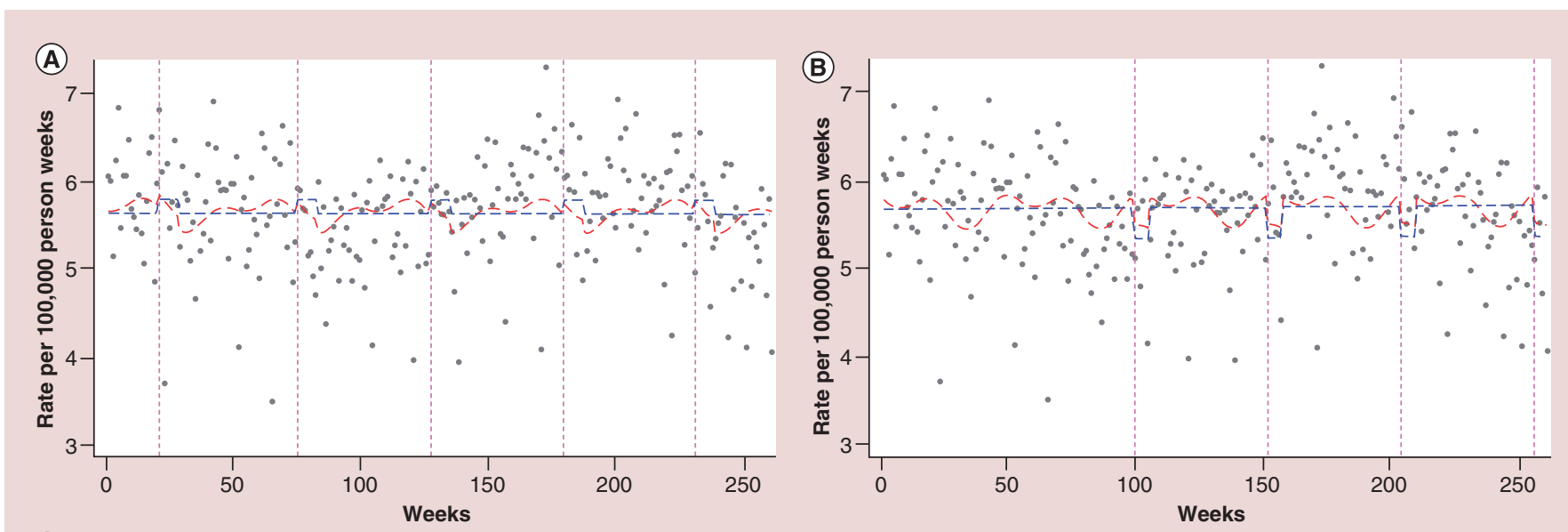

(C)

(D)
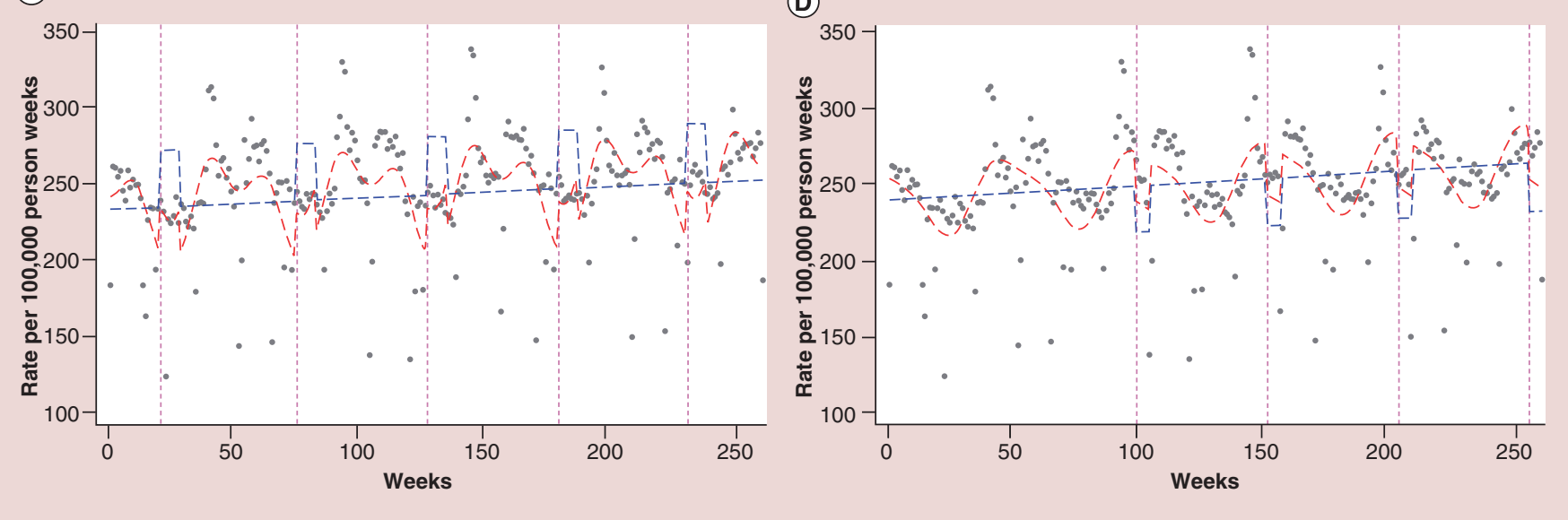

Figure 2. Scatterplots showing weekly event rates for atrial fibrillation diagnoses and pulse checks per 1000 person weeks over the study period along with predicted values from seasonality adjusted Poisson regression models. Red dashed line for atrial fibrillation diagnoses following World Heart Rhythm Week (A), atrial fibrillation diagnoses following Global Atrial Fibrillation aware week (B), pulse checks following World Heart Rhythm Week (C) and pulse check following Global Atrial Fibrillation aware week (D). De-seasonalized trends for each model are shown with the dashed blue line. Vertical dashed lines indicate the weeks in which the awareness campaigns took place.

longer term impact of the awareness campaigns in this study as it was not possible to accurately capture temporal confounders likely to impact such a hypothesis.

We considered seasonality to be the key time varying confounder that could impact the associations under study, however, should other time varying confounders exist, these may bias the results observed. We explored our hypothesis within all individuals aged greater than 18 years in order to ensure any impact of the campaigns on earlier identification of AF and its symptoms was captured, subgroup analyses exploring associations within specific subgroups of age did not identify differing results within specific subgroups. A further limitation is that we have carried out hypothesis tests on a large number of secondary outcomes in this study, the results presented must be interpreted in this context. As such random error may be a plausible explanation for the decreased rates of events observed following GAFAW, and the increased rates of events observed following WHRW, including the increased rate of pulse checks. It is also possible that not all symptoms and investigations under investigation were coded in the CPRD, and that AF diagnoses may even go unrecorded, particularly when diagnosed in secondary care. Under-recording of such events may limit the power of our study to detect increases post-awareness campaign and, if differential between baseline and postawareness campaign periods, may bias the results. It should also be noted that the 8-week period following WHRW was included in the baseline period for GAFAW and vice versa, any effect of one campaign may therefore have impact on the baseline rate used for the other campaign. 
WHRW has a strong focus on encouraging the public to 'know your pulse' and complete a 'pulse check challenge' [5]. The finding that the rate of pulse checks is increased following WHRW therefore suggests that these activities may be having some success in producing a transient increase in public interest in having their GP perform a pulse check. Alternatively, it may be raising awareness within the GP community, resulting in an increased level of promotion of pulse checks by GPs during consultations. There may be a number of reasons an increase in pulse checks does not translate into an immediate, transient 8-week increase in the rate of AF diagnoses. Notably, an increase of $16 \%$ in the rate of pulse checks may not translate into a large enough increase in the identification of $\mathrm{AF}$ cases for us to be able to detect it in this study.

As outlined above, the decreased rate of consultations in which symptoms of AF were recorded following GAFAW may be due to random or systematic error. However, should this not be the case, a possible mechanism by which these associations may have been observed could be that individuals with these symptoms had their pulse checked at ad-hoc or 'pop-up' health promotion events and received reassurance that they did not have an abnormal pulse leading them to choose not to present at their GP with these symptoms. The decreased rate of pulse checks recorded by GPs in the 8 weeks following GAFAW is consistent with this. We would note that this contrasts with the justification provided above for the results observed for WHRW therefore while we offer both as possible explanations for the results observed, we believe it is unlikely that the two campaigns have such contrasting effects given their similarities.

Our inability to identify a substantial impact of awareness campaigns on AF diagnosis rates suggests that awareness campaigns of this nature alone will not increase the rate of diagnosis of AF sufficiently to produce a substantial decrease in the prevalence of silent $\mathrm{AF}$ and the potential morbidity and healthcare burden associated with it [13-16]. Targeted or opportunistic screening programs represent a far more costly alternative to awareness campaigns, but would produce meaningful improvements in the identification of silent AF [17]. The UK National Screening Committee was scheduled to reconsider their recommendation against systematic population screening for AF in 2017/2018. This reconsideration process remains ongoing [18]. Should population screening for AF not be recommended, our results suggest $\mathrm{AF}$ awareness campaigns may require greater resources in order to be effective in increasing the identification of AF.

In conclusion, the rate of pulse checks was increased by $16 \%$ in the 8 weeks following WHRW; however, this did not translate into a detectable increase in the rate of AF diagnoses, identification of AF-related symptoms or AF-related prescriptions over the same time frame. These findings suggest that awareness campaigns do not bring about short-term increases in the rates of AF diagnoses and that, in the absence of population screening programs for AF, the scope and resources of such awareness campaigns may need to be increased to bring about substantial increases in the rates of AF diagnoses.

\section{Future perspective}

Our finding, that a short-term increase in the rate of diagnoses of AF is not observed in the 8 weeks following existing $\mathrm{AF}$ awareness campaigns, suggests that while the campaigns may raise awareness about $\mathrm{AF}$, they do not have an acute or short-term impact on the frequency of diagnoses. Further research is required to establish whether the awareness campaigns impact the rate of diagnoses in a different manner. However, should such studies substantiate our findings, it would point to a need to introduce alternative approaches to support the identification of silent and symptomatic AF.

\section{Supplementary data}

To view the supplementary data that accompany this paper please visit the journal website at: www.futuremedicine.com/doi/full/ 10.2217/fca-2019-0025

\section{Author contributions}

V Allan, SV Ramagopalan and CJ Sammon designed the study, CJ Sammon was responsible for data management and analysis. All authors were involved in the interpretation of results and the drafting of the manuscript. 
Financial \& competing interests disclosure

This study was funded by Bristol-Myers Squibb (BMS) and Pfizer. V Allan, L McDonald, F Mehmud and SV Ramagopalan are employees of BMS. CJ Sammon is a full-time employee of PHMR, PHMR received financial support for the work from Bristol-Myers Squibb and Pfizer. The authors have no other relevant affiliations or financial involvement with any organization or entity with a financial interest in or financial conflict with the subject matter or materials discussed in the manuscript apart from those disclosed.

No writing assistance was utilized in the production of this manuscript.

\section{Ethical conduct of research}

This study was approved by the Independent Scientific Advisory Committee (ISAC) with CPRD number 18_146.

\section{Data sharing}

CPRD data were provided under a licence that does not permit sharing. Data are, however, obtainable directly from CPRD under their standard conditions.

\section{Open access}

This work is licensed under the Attribution-NonCommercial-NoDerivatives 4.0 Unported License. To view a copy of this license, visit http://creativecommons.org/licenses/by-nc-nd/4.0/

\section{Summary points}

- Individuals with undiagnosed atrial fibrillation (AF) are unknowingly at an increased risk of experiencing fatal and disabling strokes.

- AF awareness campaigns, such as the annually held, World Heart Rhythm Week (WHRW) and Global AF aware week (GAFAW) may help to accelerate the diagnosis and treatment of individuals with undiagnosed AF.

- However, to date, there are no published quantitative evidence exploring the effectiveness of such campaigns.

- We used the UK Clinical Practice Research Datalink to assess whether rates of AF-related consultations changed in the 8 weeks following WHRW and GAFAW.

- While the rate of pulse checks was increased following WHRW, the rate of AF diagnoses was not increased following either awareness campaign.

- The findings highlight the need for consideration of alternative approaches to support the identification of silent and symptomatic AF.

\section{References}

Papers of special note have been highlighted as: • of interest; $\bullet \bullet$ of considerable interest

1. Sposato LA, Cipriano LE, Saposnik G, Ruíz Vargas E, Riccio PM, Hachinski V. Diagnosis of atrial fibrillation after stroke and transient ischemic attack: a systematic review and meta-analysis. Lancet. Neurol. 14(4), 377-387 (2015).

2. Ganapathy AV, Monjazeb S, Ganapathy KS, Shanoon F, Razavi M. 'Asymptomatic' persistent or permanent atrial fibrillation: a misnomer in selected patients. Int. J. Cardiol. 185, 112-113 (2015).

3. Moran PS, Teljeur C, Ryan M, Smith SM. Systematic screening for the detection of atrial fibrillation. Cochrane Database Syst. Rev.(3), CD009586 (2016).

4. Kirchhof P, Benussi S, Kotecha D et al. 2016 ESC Guidelines for the management of atrial fibrillation developed in collaboration with EACTS. Eur. Heart J. 37(38), 2893-2962 (2016).

5. World Heart Rhythm Week - Arrhythmia Alliance (2019). www.heartrhythmalliance.org/aa/uk/heart-rhythm-week

- Details one of the awareness campaigns under investigation.

6. Wendelboe AM, Raskob GE, Angchaisuksiri P et al. Global public awareness about atrial fibrillation. Res. Pract. Thromb. Haemost. 2(1), 49-57 (2018).

7. Chandratheva A, Lasserson DS, Geraghty OC, Rothwell PM; Oxford Vascular Study. Population-based study of behavior immediately after transient ischemic attack and minor stroke in 1000 consecutive patients: lessons for public education. Stroke 41(6), 1108-1114 (2010).

8. Herrett E, Gallagher AM, Bhaskaran K et al. Data Resource Profile: Clinical Practice Research Datalink (CPRD). Int. J. Epidemiol. 44(3), 827-836 (2015).

9. Bernal JL, Cummins S, Gasparrini A. Interrupted time series regression for the evaluation of public health interventions: a tutorial. Int. J. Epidemiol. 46(1), 348-355 (2017).

-• Accessible introduction to interrupted time series methodology. 
10. AF Association Global AF Aware Week (2018). www.heartrhythmalliance.org/afa/uk/af-aware-week

- Details one of the awareness campaigns under investigation.

11. Ruigómez A, Johansson S, Wallander MA, Rodríguez LAG. Incidence of chronic atrial fibrillation in general practice and its treatment pattern. J. Clin. Epidemiol. 55(4), 358-363 (2002).

12. Bhaskaran K, Gasparrini A, Hajat S, Smeeth L, Armstrong B. Time series regression studies in environmental epidemiology. Int. J. Epidemiol. 42(4), 1187-1195 (2013).

13. Healey JS, Connolly SJ, Gold MR et al. Subclinical atrial fibrillation and the risk of stroke. N. Engl. J. Med. 366(2), 120-129 (2012).

14. Flaker GC, Belew K, Beckman K et al. Asymptomatic atrial fibrillation: demographic features and prognostic information from the Atrial Fibrillation Follow-up Investigation of Rhythm Management (AFFIRM) study. Am. Heart J. 149(4), 657-663 (2005).

15. Turakhia MP, Shafrin J, Bognar K et al. Economic burden of undiagnosed nonvalvular atrial fibrillation in the United States. Am. J. Cardiol. 116(5), 733-739 (2015).

16. Savelieva I, Camm AJ. Clinical relevance of silent atrial fibrillation: prevalence, prognosis, quality of life, and management. J. Interv. Card. Electrophysiol. 4(2), 369-382 (2000).

17. Welton NJ, McAleenan A, Thom HH et al. Screening strategies for atrial fibrillation: a systematic review and cost-effectiveness analysis. Health Technol. Assess. (Rockv.) 21(29), 1-236 (2017).

18. UK National Screening Committee (NSC). Atrial fibrillation (2019). https://legacyscreening.phe.org.uk/atrialfibrillation 